Bull. Mater. Sci., Vol. 17, No. 6, November 1994, pp. 1057-1064. (C) Printed in India.

\title{
Influence of deviation from stoichiometry on the photoluminescence in CdTe doped with indium
}

\author{
SUMA GURUMURTHY, K S R K RAO, A K SREEDHAR, H L BHAT, \\ B SUNDERSHESHU*, R K BAGAI* and VIKRAM KUMAR* \\ Department of Physics, Indian Institute of Science, Bangalore 560012 , India \\ *Solid State Physics Laboratory, Lucknow Road, Delhi 110 054, India
}

\begin{abstract}
Low temperature photoluminescence of vacuum and cadmium annealed CdTe:In is reported here. A new peak at $\sim 1.14 \mathrm{eV}$ related to transitions from the conduction band to an acceptor involving a tellurium vacancy has been observed.
\end{abstract}

Keywords. Cadmium telluride; photoluminescence; annealing; stoichiometry; indium doping.

\section{Introduction}

Cadmium telluride is an important substrate material used in the advanced $\mathrm{Hg}_{1-x} \mathrm{Cd}_{x} \mathrm{Te}(\mathrm{MCT})$ programme. It is important not only as a substrate for epitaxy, but also as an active part of potential heterojunction device structures, in the fabrication of nuclear detectors, solar cells, etc. All these applications are very sensitive to the purity of samples, particularly, if cadmium telluride is to be used as a substrate for epitaxial growth of MCT, since outdiffusion of the impurities into the epilayer during growth and subsequent annealing will impair the performance of MCT detector. Many recent investigations, designed to identify various types of acceptor impurities, have employed nondestructive techniques such as EPR and low temperature photoluminescence spectroscopy (Molva et al 1982).

Although, photoluminescence spectroscopy is a sensitive, nondestructive technique that can provide valuable information concerning the type and distribution of defects and impurities in a crystal, the interpretation of PL data in II-VI semiconductors is not straight forward because of the complex nature of the defects introduced by deviations from stoichiometry and the interaction of these defects with the dopant atoms. Hence, the results obtained by different groups do not necessarily agree and some interpretations are contradictory.

Self-compensation phenomena in halogen doped cadmium telluride has been extensively studied by Bell et al (1974). Indium (a donor) is often intentionally introduced into CdTe for the purpose of compensating native defects and producing semi-insulating material. The present understanding of defects in $\mathrm{CdTe}$ is rather meagre. Definitive information on the microscopic structure of native defects is lacking.

Most PL studies on CdTe have been restricted to the energy range above 1.24 $\mathrm{eV}$. The commonly observed features in the $4.2 \mathrm{~K}$ PL spectra of CdTe are a series of sharp peaks in the range $1.5-1.6 \mathrm{eV}$ attributed to excitonic and near band edge emissions, $1.42 \mathrm{eV}$ band with phonon replicas and a broad band around $1.1 \mathrm{eV}$. There have been only a few reports on the $1.1 \mathrm{eV}$ and lower energy bands. The $1.1 \mathrm{eV}$ band in bulk CdTe samples as reported by a few groups is thought to be 
due to transitions from a deep level formed (de Nobel 1959; Lorentz and Segall 1963; Vavilov et al 1967; Das et al 1990) as a result of deviation from stoichiometry. But, regarding the $1.42 \mathrm{eV}$ defect peak there has been a lot of controversy, with different groups assigning different transitions to it (Vavilov et al 1967; Rud et al 1971; Das et al 1990; Babentsov et al 1992). Despite the prominence of 1.42 $\mathrm{eV}$ bands and their long history of observation, the possible origin of this band still remains a matter of dispute and conjecture.

Heat treatments under tellurium or cadmium overpressure are of particular interest because the overall stoichiometry of the crystal can be changed. Suitable conditions for the observation of PL spectra can be achieved, for example, by brief thermal annealing in vapours of the components of the semiconductor. In this paper we report the $4.2 \mathrm{~K} \mathrm{PL}$ of vacuum and cadmium annealed $\mathrm{CdTe}$ doped with indium and the presence of a new peak related to a tellurium vacancy.

\section{Experimental}

Indium doped CdTe crystals, grown by the asymmetrical Bridgman method (Bagai and Borle 1989) have been used in the present investigation. In this method, the growth ampoule is held asymmetrically inside a cylindrical furnace and crystals are grown by lowering the ampoule vertically through the temperature gradient of about $10^{\circ} \mathrm{C} \mathrm{cm}^{-1}$ at a speed of $0.5 \mathrm{~mm} \mathrm{~h}^{-1}$. Single crystal wafers of CdTe:In oriented along $\langle 111\rangle$ directions were cut from the grown ingot from which samples of required dimensions were prepared. Typical sample size was $3 \times 5 \mathrm{~mm}^{2}$. All the cut samples were chemically etched in $1 \%$ bromine in methanol for $5 \mathrm{~min}$ followed by rinsing for $1 \mathrm{~h}$ in methanol to remove traces of bromine. After this, the samples were treated in $1 \mathrm{~N} \mathrm{KOH}$ in methanol for $5 \mathrm{~min}$ to remove the tellurium film resulting in the bromine methanol etch (Amirtharaj and Pollak 1984).

Cadmium annealing was done by placing the CdTe sample in a sealed evacuated dumb bell shaped ampoule in presence of an excess cadmium vapour pressure. The cadmium vapour atmosphere was created by placing a suitable amount of high purity $(6 \mathrm{~N})$ cadmium in the ampoule at the same temperature as the CdTe sample. Vacuum annealing was done by placing CdTe sample in a sealed evacuated quartz ampoule without cadmium. Both the annealings were carried out at $650^{\circ} \mathrm{C}$ for $6 \mathrm{~h}$. Samples were quenched immediately after annealing in liquid nitrogen. A piece of the original sample which was taken from the adjacent part of the wafer and which was not annealed was kept as a control sample (for the sake of comparison).

Photoluminescence measurements were carried out at $4.2 \mathrm{~K}$ using MIDAC Fourier Transform PL system. An Argon ion laser operating at a wavelength of $5145 \AA$ was used as a source of excitation. The exposed sample area was $\cong 3 \mathrm{~mm}^{2}$. Neutral density filters were used to record the spectra at high laser powers. PL signal was detected by a $\mathrm{LN}_{2}$ cooled Ge-photodetector whose operating range is about $0.75-1.9 \mathrm{eV}$. Measurements were carried out with a resolution of $0.5 \mathrm{meV}$.

\section{Results and discussion}

PL measurements were performed on all the pieces cut from a single CdTe:In wafer, prior to annealing treatments. PL spectra recorded were essentially the same 
both in terms of energy position and intensity of the spectral lines. Therefore, any changes which occur in the PL spectra after annealing treatments could be attributed to the changes caused by annealing and not to differences in the starting materials. A typical photoluminescence spectrum of CdTe:In is shown in figure 1. The main features as can be seen are the $1 \cdot 1 \mathrm{eV}$ broad band (attributed to cadmium vacancies), $1.4 \mathrm{eV}$ peak with phonon replicas (controversial band) and the $1.54 \mathrm{eV}$ edge emission.

Figure 2 shows the PL spectra of the $1.11 \mathrm{eV}$ broad band of the control, vacuum and cadmium annealed samples recorded at $4.2 \mathrm{~K}$ at a laser excitation power of $100 \mathrm{~mW}$. It is clearly evident from spectra (a) and (b) in this figure that there is a 15 fold increase in the peak height and approximately 8 times increase in the integrated intensity on vacuum annealing. Table I gives the integrated intensity for

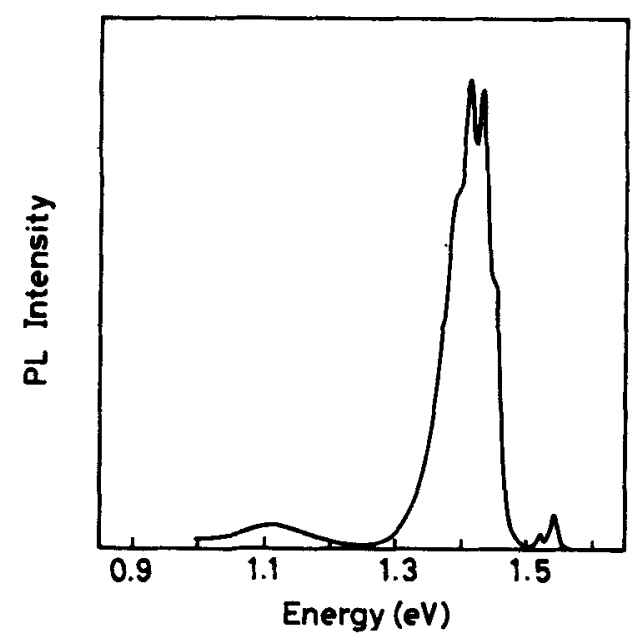

Figure 1. PL spectrum of CdTe:In at $4.2 \mathrm{~K}$ in the range $0.9-1.6 \mathrm{eV}$.

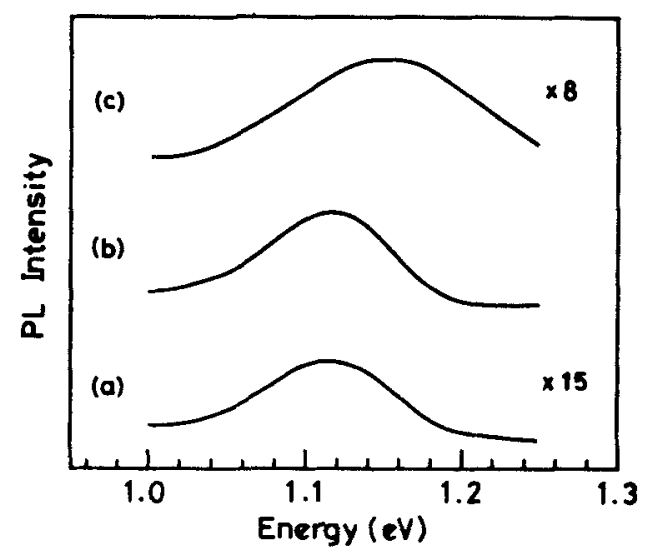

Figure 2. PL spectra at $4.2 \mathrm{~K}$ showing the $1.11 \mathrm{eV}$ band in (a) control. (b) vacuum annealed and (c) cadmium annealed CdTe:In samples (Excitation power: $100 \mathrm{~mW}$ ). 
various peaks in the control and annealed samples. However, this peak as seen from spectrum (c) is found to have disappeared in the sample annealed in a cadmium atmosphere. This suggests that the band is related to transitions from a native defect involving a cadmium vacancy. Vavilov et al (1967) attributed this band to electron transitions from the doubly charged state of the double acceptor at $\left(E_{\mathrm{c}}-0.6 \mathrm{eV}\right)$ to the valence band. Sobiesierski et.al (1988) have shown that the PL bands at around $1 \cdot 11 \mathrm{eV}$ in chemically etched bulk samples are a result of deviation from stoichiometry. However, the few reports by Kernocker et al (1985) and Bowman and Cooper (1988) suggest the band around $1.11 \mathrm{eV}$ to arise from some iron impurity.

Figure 3 shows the PL spectra of $1.4 \mathrm{eV}$ band of the control, vacuum and cadmium annealed samples recorded at $4.2 \mathrm{~K}$ at $100 \mathrm{~mW}$ laser power. This band which occurs independent of growth technique has not been identified and its origin is therefore widely discussed. This band has the zero phonon line at $1.453 \mathrm{eV}$. The peak intensity of this band in vacuum annealed sample decreased by approximately 5 times and about 1.5 times in cadmium annealed sample. However, the integrated intensity of this $1.4 \mathrm{eV}$ band in the vacuum annealed sample as can be seen from table 1 , decreased by $\sim 1.3$ times and increased by $\sim 1.5$ times in the cadmium sample when compared to the control sample. A shift of the peak energy of $\sim 4 \mathrm{meV}$ for a decade change in the excitation intensity can be seen from figures $4 \mathrm{a}$

Table 1. Integrated intensity of the PL peaks of the control and annealed samples.

\begin{tabular}{lcccc}
\hline & $\begin{array}{c}\text { Laser power } \\
\text { Sample }\end{array}$ & \multicolumn{3}{c}{ Integrated intensity (au) } \\
\cline { 3 - 5 } & $(\mathrm{mW})$ & $1.116 \mathrm{eV}$ & $1.146 \mathrm{eV}$ & $1.4 \mathrm{eV}$ \\
\hline Control & 100 & 5 & - & 21 \\
$\begin{array}{l}\text { Vacuum } \\
\text { annealed }\end{array}$ & 100 & 39 & - & 16 \\
$\begin{array}{c}\text { Cadmium } \\
\text { annealed }\end{array}$ & 100 & - & 9 & 35 \\
\hline
\end{tabular}

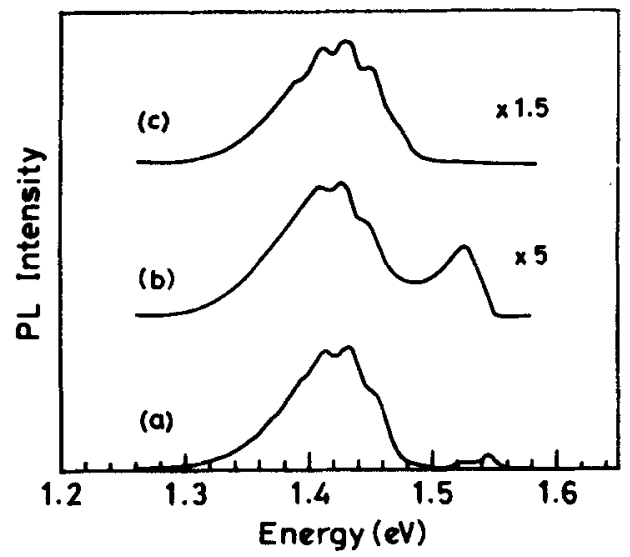

1. «цure 3. PL spectra at $4.2 \mathrm{~K}$ showing the $1+4 \mathrm{cV}$ band in (a) control, (b) vacuum annealed and (c) cadmium annealed samples. (Excitation power: $100 \mathrm{~mW}$ ). 

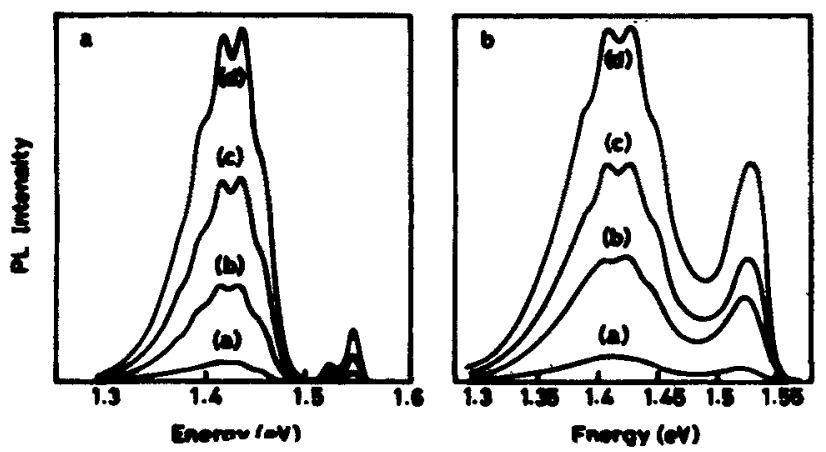

Figure 4. PL spectra at $4.2 \mathrm{~K}$ recorded at different excitation intensities of (a) control and (b) vacuum annealed samples. (Excitation intensities: (a) $10 \mathrm{~mW}$, (b) $50 \mathrm{~mW}$, (c) $150 \mathrm{~mW}$ and (d) $250 \mathrm{~mW}$ ).

and $b$ for the control and vacuum annealed samples respectively. This shift suggests this band to involve a D-A transition, which is in agreement with earlier reports (Rud et al; 1971, Das et al 1990; Babentsov et al 1992).

The transition responsible for this band in all CdTe crystals has been interpreted in various ways. According to de Nobel (1959) the band has been attributed to C-A like transitions. D-A transition has been one of the other interpretation by various groups (Rud et al 1971; Das et al 1990; Babentsov et al 1992). There has been a lot of speculation regarding the donors and acceptors involved in the transition. Some groups have assigned this band to transition from shallow donors to deep acceptors involving a cadmium vacancy (Vavilov et al 1967), while others to transition from donors formed by interstitial cadmium atoms to acceptors (Vavilov et al 1967; Rud et al 1971). Our results on the effect of cadmium vapour pressure on the luminescence intensity make it difficult to attribute the $1.4 \mathrm{eV}$ band to cadmium vacancy. D-A complex of the type $C d_{i}-X$ may possibly be involved in this transition. However, further investigations must be carried out before any conclusion can be drawn. Investigations by Hofmann et al (1992) clearly show that the emission in this spectral range consists of (at least) three independent emission bands, the first one located at $1.45 \mathrm{eV}$ involving a D-A transition (Chamonal et al 1982), the second one of unknown origin at $1.475 \mathrm{eV}$ (Onodera and Taguchi 1990 ) and the third one at $1.478 \mathrm{eV}$ (Hofmann et al 1992) arising from a D-A recombination involving the A-centres. It should be noted that all these bands have completely different origins and unfortunately interfere in most of the samples leading to considerable discrepancy.

The edge emission at $1.544 \mathrm{eV}$ as seen from figures 3 (spectrum a) and $4 \mathrm{a}$ is attributed to a $\mathrm{C}-\mathrm{A}$ transition as the peak energy does not vary with excitation intensity (Agrinskaya et al 1971; Barneso and Zanio 1975). The acceptor involved is a singly ionized cadmium vacancy. The hump seen at $1.523 \mathrm{eV}$ is the $\mathrm{LO}$ phonon replica of the $1.544 \mathrm{eV}$ peak. Excitation intensity dependence of the peak energy can be seen in figure $4 a$.

However, the edge emission at $1.528 \mathrm{eV}$ as seen from figures 3 (spectrum b) and $4 \mathrm{~b}$ is a D-A transition as the peak energy shifted by $\sim 4 \mathrm{meV}$ per decade 
change in the excitation intensity (Agrinskaya et al 1971). The acceptor is a cadmium vacancy with an ionization energy of $60 \mathrm{meV}$ as reported earlier. It can be seen from spectrum (c) in figure 3 that the edge emissions have disappeared in the cadmium annealed sample.

Spectrum (c) in figure 2 shows a peak at $\sim 1.146 \mathrm{eV}$. A level at $E_{\mathrm{v}}+0.46 \mathrm{eV}$ corresponding to a tellurium vacancy has been reported in the literature (Caillot 1972). Halsted et al (1961) for CdTe and Uchida (1964) for $\mathrm{ZnS}$ and CdS have shown that a cation deficiency results from firing in an over-pressure of the anion and vice versa. This shows that firing the sample in an over-pressure of cadmium has resulted in the formation of tellurium vacancies and hence the $1.146 \mathrm{eV}$ peak can be attributed to $\mathrm{C}-\mathrm{A}$ like transitions involving the tellurium vacancy. To confirm the origin of this peak in our spectrum, the cadmium annealed sample was again subjected to a further annealing at $650^{\circ} \mathrm{C}$ for $6 \mathrm{~h}$, but in a saturated tellurium atmosphere. Figure 5 shows the PL spectra of the control, cadmium annealed and subsequent tellurium annealed samples. The disappearance of the $1.146 \mathrm{eV}$ peak and reappearance of the $1.116 \mathrm{eV}$ peak after tellurium annealing confirms that the $1.146 \mathrm{eV}$ peak is to be related to transitions involving tellurium vacancy. No shift of this peak with excitation intensity was observed. The decrease in the peak intensity of the $1.4 \mathrm{eV}$ band in the tellurium annealed sample as compared to the cadmium annealed one (figure 6), suggests that the $1.4 \mathrm{eV}$ band does not involve a cadmium vacancy. So, firing of the sample in a tellurium atmosphere has resulted in the formation of cadmium vacancies, which is confirmed by the reappearance of the $1.116 \mathrm{eV}$ band and the decrease in the intensity of the $1.4 \mathrm{eV}$ band.

The edge emission at $1.532 \mathrm{eV}$ as seen from figure 6 (spectrum b) is again a D-A like transition with $\sim 10 \mathrm{meV} /$ decade shift in the peak energy. A large shift of this magnitude and more has been seen in compensated GaAs (Yu 1977; Swaminathan et al 1981a) and AlGaAs (Mazzaschi et al 1980; Swaminathan et al 1981b) and in moderately dopcel InP (Swaminathan et al 1985). Such a large shift with excitation can happen cilh', when the material is closely compensated or if

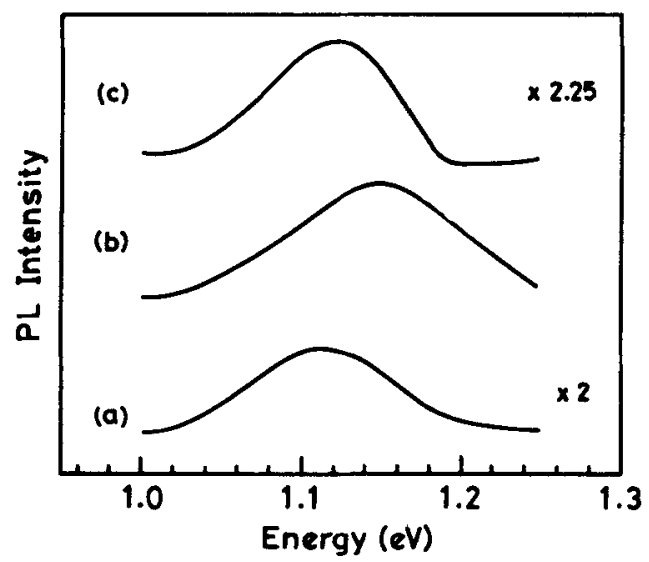

Figure 5. PL spectra at $4.2 \mathrm{~K}$ showing the $1.116 \mathrm{cV}$ and $1.146 \mathrm{eV}$ bands in (a) control, (b) cadmium annealed and (c) tellurium annealed subsequent to cadmium annealing samples. (Excitation power: $100 \mathrm{~mW}$ ). 


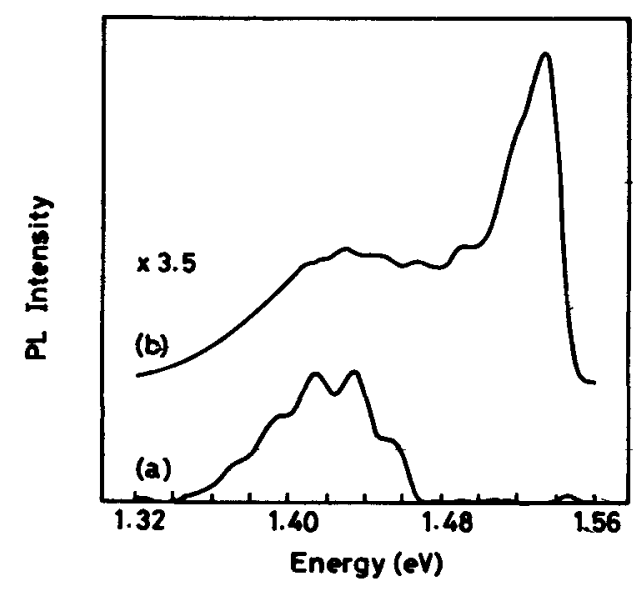

rigure 6. PL spectra at $4.2 \mathrm{~K}$ showing the $1.4 \mathrm{eV}$ band and the edge emissions in (a) cadmium annealed and (b) tellurium annealed subsequent to cadmium annealing samples. (Excitation power: $100 \mathrm{~mW}$ ).

the donor or the acceptor involved is a deep one. More experiments are needed to verify this and work in this direction is under progress.

\section{Conclusions}

Our PL results show the $1.116 \mathrm{eV}$ peak in the vacuum annealed sample to involve cadmium vacancy. The annealing experiments in cadmium and tellurium overpressure suggests that the $1.146 \mathrm{eV}$ peak in cadmium annealed sample to involve a tellurium vacancy and $1.4 \mathrm{eV}$ peak to involve transitions from shallow interstitial cadmium donors to acceptors.

\section{Acknowledgement}

We thank Dr Suman B Iyer for helpful discussions.

\section{References}

Agrinskaya N V, Arkad'eva E N and Matveev O A 1971 Soyiet Phys. Semicond. 5762

Amirtharaj P M and Pollak F H 1984 Appl. Phys. Lett. 45789

Babentsov V N, Rashkovetskii L V, Sal'kov E A and Tarbaev N I 1992 Sov. Phys. Semicond. 26608 and references therein

Bagai R K and Borle W N 1989 J. Cryst. Growth 94561

Barnes C E and Zanio K 1975 J. Appl. Phys. 463959

Bell R O, Wald F V, Canali C, Nava F and Ottaviani G 1974 IEEE Trans. Nucl. Sci. NS-21 331

Bowman Jr R C and Cooper D E 1988 Appl. Phys. Lett. 531521

Caillot M 1972 Phys. Lett. A38 2

Chamonal J P, Molva E and Pautrat J L 1982 Solid State Commun. 43801

Das S R, Cook J G, Rowell N L and Aouadi M S $1990 \mathrm{~J}$. Appl. Phys. 685796 and references therein Halsted R E, Lorenz M R and Segall B 1961 J. Phys. Chem. Solids 22109 
Hofmann D M, Omling P, Grimmeiss H G, Meyer B K, Benz K W and Sinerius D 1992 Phys. Rev. B45 6247

Kemocker R, Lischka K and Palmetshofer L 1988 J. Cryst. Growth 86625

Lorentz M R and Segall B 1963 Phys. Lett. 718

Mazzaschi J, Barrau J, Brabant J C, Brousseau M, Maaref H, Voillot F and Boissy M C 1980 Rev. Phys. Appl. 15861

Molva E, Chamonal J P and Pautrat J L 1982 Phys. Status Solidi B109 635

de Nobel 1959 Philips Res. Rep. 14361

Onodera C and Taguchi T $1990 \mathrm{~J}$. Cryst. Growth 101502

Rud Yu V, Sanin K V and Shreter Yu G 1971 Sov. Phys. Semicond. 5573

Sobiesierski Z, Dharmdasa I M and Williams R H 1988 Appl. Phys. Lett. 532623

Swaminathan V. Schumaker $N$ E and Zilko J L 1981 a J. Lumin. 22153

Swaminathan V, Anthony P J, Zilko J L, Sturge M D and Schumaker N E 1981b J. Appl. Phys. 52 5603

Swaminathan V, Donnelly V M and Long J 1985 J. Appl. Phys. 584565

Uchida I 1964 J. Phys. Soc. Jin 19670

Vavilov V S, Gippius A A and Panossian J R 1967 Proc. int. conf. II-VI semiconducting compounds (ed.) D G Thomas (New York: Benjamin Inc.) p. 743

Yu P W 1977 J. Appl. Phys. 485043 